\title{
Excellent Extensions and Ding Projective Modules
}

\author{
Juxiang $\operatorname{Sun}^{1}$ \\ ${ }^{1}$ School of Mathematics and Information Science, Shangqiu Normal University, Shangqiu, China \\ Correspondence: Juxiang Sun, School of Mathematics and Information Science, Shangqiu Normal University, \\ Shangqiu, China. E-mail: sunjx86@163.com
}

Received: November 27, 2013 Accepted: January 11, 2014 Online Published: May 13, 2014

doi:10.5539/jmr.v6n2p100 URL: http://dx.doi.org/10.5539/jmr.v6n2p100

\begin{abstract}
In this paper, we study the invariant properties of global Ding projective dimensions under excellent extensions of rings. And the excellent extensions of Ding-Chen rings are also discussed.
\end{abstract}

Keywords: excellent extension, Ding-Chen ring, Ding projective module

\section{Introduction}

In studying the algebraic structure of group rings, Passman (1977) introduced the notion of the excellent extensions of rings (the name comes from Bonami, 1984). Such extensions of rings are vital because they include two important classes of rings, that is, finite matrix rings and skew group rings $R G\left(|G|^{-1} \in R\right)$. Many authors have studied the invariant properties of rings under excellent extensions (see e.g. Bonami, 1984; Feng, 1997; Passman, 1977; Xue, 1996; Xiao, 1994). It has been known that many important homological properties, such as the (weak) global dimension of rings, the projectivity, injectivity and flatness of modules and so on, are invariant under excellent extensions (Xue, 1996).

Throughout this paper, all rings are associative with identity and all modules are unitary. $M_{R}\left({ }_{R} M\right)$ denotes a right (left) $R$-module, and we freely use the terminology and notations of (Boami, 1984; Ding \& Chen, 1993). A right $R$ module $M$ is called $F P$-injective in (Stenström, 1970) if $\operatorname{Ext}_{R}^{1}(N, M)=0$ for all finitely presented right $R$-modules $N$. The FP-injective dimension of $N$, denoted $\mathrm{FP}_{-i d} N$, is defined similarly to the classical injective dimension. A ring $R$ is called an $n-F C$ ring if $R$ is a left and right coherent ring with $\mathrm{FP}_{-i d} R \leq n$ and $\mathrm{FP}-\mathrm{id} R_{R} \leq n$. These rings were introduced and studied by Ding and Chen $(1993,1996)$. Gillespie (2010) first called a ring Ding-Chen when it is $n$-FC for some $n$, which seen to have many properties similar to $n$-Gorenstein rings.

As a particular case of Gorenstein projective modules, Ding projective modules was introduced by Gillespie (2010) and further studied by many authors (see e.g., Wang \& Liu; Yang, 2012, 2013). An $R$-module $M$ is called Ding projective if there exists an exact sequence of projective $R$-modules

$$
\cdots \rightarrow P_{1} \rightarrow P_{0} \rightarrow P^{0} \rightarrow P^{1} \rightarrow \cdots
$$

with $M=\operatorname{Ker}\left(P^{0} \rightarrow P^{1}\right)$ and which remains exact after applying $\operatorname{Hom}_{R}(-, F)$ exact for each flat $R$-module $F$. This class of modules have been treated by different authors.

In this paper, we will study the excellent extension of Ding-Chen rings and global Ding projective dimensions of rings under excellent extensions.

In Section 2, we give some notations in our terminology and some preliminary results which are often used in this paper. In Section 3, we prove our main results.

Theorem 1.1 Let $S \geq R$ be an excellent extension, then $S$ is a Ding-Chen ring if and only if so is $R$.

We used r.Dpd ${ }_{R} M$ to denoted the right Ding projective dimension of a module $M$ in $\operatorname{Mod} R$, which defined as the smallest non-negative $n$ such that there exists an exact sequence

$$
0 \rightarrow D_{n} \rightarrow D_{n-1} \rightarrow \cdots \rightarrow D_{1} \rightarrow D_{0} \rightarrow M \rightarrow 0
$$

with each $D_{i}$ Ding projective. We used r.gl.Dpd $S$ to denoted the right global Ding projective dimension of $R$, 
which defined as

$$
\text { r. } \operatorname{gl} . \operatorname{Dpd}(S)=\sup \{\mathrm{r} . \operatorname{Dpd}(M) \mid M \in \operatorname{Mod} R\} .
$$

Theorem 1.2 Let $S \geq R$ be an excellent extension, then r. $\operatorname{gl} . \operatorname{Dpd}(R)=\mathrm{r} . \operatorname{gl} . \operatorname{Dpd}(S)$.

\section{Preliminaries}

We begin with the definition of excellent extensions of rings.

Definition 2.1 Let $R$ be a subring of a ring $S$, such that $S$ and $R$ have the same identity. Then $S$ is called a ring extension of $R$, and denoted by $R \leq S$. A ring extension $R \leq S$ is called an excellent extension, if

(1) $S$ is right $R$-projective (Passman, 1977, p. 273), that is, if $N_{S}$ is a submodule of $M_{S}$ and if $N_{R}$ is a direct summand of $M_{R}$, denoted by $N_{R} \mid M_{R}$, then $N_{S} \mid M_{S}$.

(2) $S$ is a free normalizing extension of $R$, that is, there exist $b_{1}, b_{2}, \cdots, b_{n}$, such that and ${ }_{R} S$ and $S_{R}$ are free with a common basis $\left\{b_{1}, b_{2}, \cdots, b_{n}\right\}$ such that $b_{i} R=R b_{i}$, for each $i$.

Example 2.2 (See Bonami, 1984; Passman, 1977)

(1) Let $R$ be a ring, then $M_{n}(R)$ (the matrix ring of $R$ of degree $n$ ) is an excellent extension of $R$.

(2) Let $R$ be a ring and $G$ a finite group. If $|G|^{-1} \in R$, then the skew group ring $R G$ is an excellent extension of $R$.

(3) Let $A$ be a finite-dimensional algebra over a field $K$, and let $F$ be a finite separable field extension of $K$. Then $A \otimes_{K} F$ is an excellent extension of $A$.

(4) Let $K$ be a field, and let $G$ be a group and $H$ a normal subgroup of $G$. If $[G: H]$ is finite and is not zero in $K$, then $K G$ is an excellent extension of $K H$.

Lemma 2.3 Let $S \geq R$ be an excellent extension. Then

(1) (Xue, 1996, Lemma 1.1) Let $M$ be an $S$-module, then $M_{S} \mid\left(M \otimes_{R} S\right)_{S}$;

(2) (Xue, 1996, Lemma 1.1) Let $N$ be a R-module, then $N_{R} \mid\left(N \otimes_{R} S\right)_{R}$;

(3) (Xiao,1994), Theorem 2.3) Let $M$ be an $S$-module, then $\operatorname{pd}_{S} M=\operatorname{pd}_{R} M=\operatorname{pd}_{S}\left(M \otimes_{R} S\right)$.

\section{Results}

Lemma 3.1 Let $S \geq R$ be an excellent extension.

(1) Let $M$ be an $S$-module, then $\mathrm{FP}-\mathrm{id}_{R} M=\mathrm{FP}-\mathrm{id}_{S} M$;

(2) Let $N$ be an R-module, then $\mathrm{FP}-\mathrm{id}_{R} N=\mathrm{FP}^{-i_{S}}\left(\operatorname{Hom}_{R}(S, N)\right)$.

Proof. (1) Assume that $\mathrm{Fp}^{-i d_{S}} M=m$, there is a finite FP-injective coresolution of $S$-module $N$ :

$$
0 \rightarrow N \rightarrow F^{0} \rightarrow F^{1} \rightarrow \cdots \rightarrow F^{m} \rightarrow 0,
$$

which also an exact sequence in $\operatorname{Mod} R$. By Mao and Ding (2005, Lemma 2.2), every $F^{i}$ is a FP-injective $R$-module. And so $\mathrm{Fp}^{-i d_{R}} N \leq m$, that is, $\mathrm{FP}-\mathrm{id}_{R} M \leq \mathrm{FP}-\mathrm{id}_{S} M$.

On the other hand, if FP-id $R=n$, there is a finite FP-injective coresolution in $\operatorname{Mod} R$

$$
0 \rightarrow M \rightarrow E^{0} \rightarrow E^{1} \rightarrow \cdots \rightarrow E^{n} \rightarrow 0 .
$$

Applying the functor $\operatorname{Hom}_{R}(S,-)$ to (3.1), there exists an exact sequence in $\operatorname{Mod} S$

$$
0 \rightarrow \operatorname{Hom}_{R}(S, M) \rightarrow \operatorname{Hom}_{R}\left(S, E^{0}\right) \rightarrow \operatorname{Hom}_{R}\left(S, E^{1}\right) \rightarrow \cdots \rightarrow \operatorname{Hom}_{R}\left(S, E^{n}\right) \rightarrow 0
$$

with $\operatorname{Hom}_{R}\left(S, E^{i}\right)$ FP-injective for each $i$, by Mao and Ding (2005, Lemma 2.3). Hence, $\operatorname{FP}_{-1 d_{S}} \operatorname{Hom}_{R}(S, M) \leq$

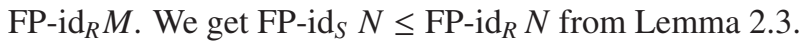

(2) The result follows from Lemma 2.1 and (1).

Theorem 3.2 If $S$ is an excellent extension of $R$, then $S$ is a Ding-Chen ring if and only if so is $R$.

Proof. "Only if" Since $R$ is a Ding-Chen ring, $\mathrm{Fp}-\mathrm{id}_{R} R=\mathrm{Fp}-\mathrm{id}_{R} R_{R}=n$ for some $n$. And we have $\mathrm{Fp}_{\mathrm{id}} S_{R}=n$,

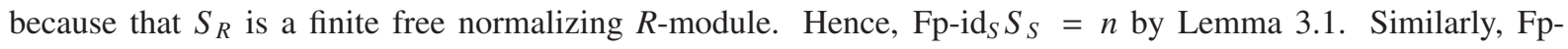
$\operatorname{id}_{S S} S=n$. Therefore, $S$ is a Ding-Chen ring. 
“If' Since $S$ is a Ding-Chen ring, FP-id ${ }_{S} S=\mathrm{FP}^{-i d_{S}} S_{S}=n$. It follows that $R$ is a Ding-Chen ring from Lemma 3.1 .

Lemma 3.3 Let $S \geq R$ be an excellent extension.

(1) For any $S$-module $N, N$ is a Ding projective $R$-module if and only if $N$ is a Ding projective $S$-module.

(2) Let $M$ be an $R$-module, then $M$ is a Ding projective $R$-module if and only if $S \otimes_{R} M$ is a Ding projective $S$-module.

Proof. Let $M$ be a Ding projective $R$-module, there is a complete projective resolution

$$
\mathcal{P}^{\prime}=\cdots \rightarrow P_{1}^{\prime} \rightarrow P_{0}^{\prime} \rightarrow P^{\prime 0} \rightarrow P^{\prime 1} \rightarrow \cdots
$$

with $M=\operatorname{Ker}\left(P^{\prime 0} \rightarrow P^{\prime 1}\right)$, which left applying the functor $\operatorname{Hom}_{R}(-, F)$ exact for any flat module $F$. Applying the functor $-\otimes_{R} S$ to $\mathcal{P}^{\prime}$, we get an exact complex $\mathcal{P}^{\prime} \otimes_{R} S$ :

$$
\mathcal{P}^{\prime} \otimes_{R} S=\cdots \rightarrow P_{1}^{\prime} \otimes_{R} S \rightarrow P_{0}^{\prime} \otimes_{R} S \rightarrow P^{\prime 0} \otimes_{R} S \rightarrow P^{\prime 1} \otimes_{R} S \rightarrow \cdots
$$

and $M \otimes_{R} S=\operatorname{Ker}\left(P^{\prime 0} \otimes_{R} S \rightarrow P^{\prime 1} \otimes_{R} S\right)$. Note that $\operatorname{Hom}_{R}(S, F)$ is a flat $R$-module for any flat $S$-module $F$ by $\left(\right.$ Feng, 1997), $\operatorname{Hom}_{R}\left(\mathcal{P}^{\prime}, \operatorname{Hom}_{S}\left({ }_{R} S_{S}, F_{S}\right)\right)$ is exact. For any right $R$-module $N_{R}$, we have the isomorphisms $\operatorname{Hom}_{R}\left(N_{R}, \operatorname{Hom}_{S}(S, F)\right) \cong \operatorname{Hom}_{S}\left(N \otimes_{R} S, F\right)$, and so $\operatorname{Hom}_{S}\left(\mathcal{P}^{\prime} \otimes_{R} S, F_{S}\right)$ is exact. Hence, $M \otimes_{R} S$ is a Ding projective $S$-module.

(1) Assume that $N_{S}$ is a Ding projective module. There is an exact sequence

$$
\mathcal{P}_{S}=\cdots \rightarrow P_{1} \rightarrow P_{0} \rightarrow P^{0} \rightarrow P^{1} \rightarrow \cdots
$$

of projective $S$-modules such that $N=\operatorname{Ker}\left(P^{0} \rightarrow P^{1}\right)$, which left applying the functor $\operatorname{Hom}_{S}(-, F)$ exact for any flat $S$-module $F$. Clearly,

$$
\mathcal{P}_{R}=\cdots \rightarrow P_{1} \rightarrow P_{0} \rightarrow P^{0} \rightarrow P^{1} \rightarrow \cdots
$$

is an exact sequence in $\operatorname{Mod} R$. We claim that $\operatorname{Hom}_{R}\left(\mathcal{P}_{R}, Q\right)$ is exact for any flat $R$-module $Q$. In fact, $\operatorname{Hom}_{R}\left({ }_{S} S_{R}, Q_{R}\right)$ is a flat $S$-module by Feng (1997), and so $\operatorname{Hom}_{S}\left(P_{S}, \operatorname{Hom}_{R}\left({ }_{S} S_{R}, Q_{R}\right)\right)$ is exact. For any $S$-module $X_{S}$, we have isomorphisms

$$
\operatorname{Hom}_{R}\left(X_{R}, Q_{R}\right) \cong \operatorname{Hom}_{R}\left(X \otimes_{S} S, Q\right) \cong \operatorname{Hom}_{S}\left(X, \operatorname{Hom}_{R}(S, Q)\right),
$$

and we get our claim. It follows that $M_{R}$ is a Ding projective module.

Conversely, assume that $M_{R}$ is Ding projective, then $M \otimes_{R} S$ is a Ding projective $S$-module. By Lemma 2.3, $M_{S} \mid\left(M \otimes_{R} S\right)_{S}$. And we get that $M_{S}$ is a Ding-projective module from (Yang, 2013, Lemma 3.2).

(2) We have only to show that $M_{R}$ is a Ding projective module if $M \otimes_{R} S$ is a Ding projective $S$-module. By (1), $\left(M \otimes_{R} S\right)_{R}$ is Ding projective. And this result follows from Lemma 2.3 and (Yang, 2013, Lemma 3.2).

Theorem 3.4 Let $S \geq R$ be an excellent extension, then r. $\operatorname{gl} . \operatorname{Dpd}(S)=\mathrm{r} . \operatorname{gl} . \operatorname{Dpd}(R)$.

Proof. Suppose that r. gl. $\operatorname{Dpd}(R)=m<\infty$. Let $M_{S}$ be a non-zero $S$-module, then r. $\operatorname{Dpd} M_{R} \leq m$. So there exists a Ding projective resolution of $M_{R}$ :

$$
0 \rightarrow F_{k} \rightarrow \cdots \rightarrow F_{1} \rightarrow F_{0} \rightarrow M \rightarrow 0
$$

with each $F_{i}$ Ding projective and $k \leq m$. By Lemma 3.3, every $F_{i} \otimes_{R} S$ is a Ding projective $S$-module. Applying the functor $-\otimes_{R} S$ to (3.2), we have an exact sequence

$$
0 \rightarrow F_{k} \otimes_{R} S \rightarrow \cdots \rightarrow F_{1} \otimes_{R} S \rightarrow F_{0} \otimes_{R} S \rightarrow M \otimes_{R} S \rightarrow 0
$$

By Lemma 2.3, $M_{S} \mid\left(M \otimes_{R} S\right)_{S}$, and hence r. $\operatorname{Dpd}_{S} M \leq m$. It follows r. $g 1 . \operatorname{Dpd}(S) \leq m$, i.e. $\operatorname{r.gl} \operatorname{Dpd}(S) \leq$ r. $\operatorname{gl} \operatorname{Dpd}(R)$.

Conversely, assume that r. $g l . \operatorname{Dpd}(S)=n$. Let $N_{R}$ be a non-zero $R$-module, then $N \otimes_{R} S \in \operatorname{Mod} S$. The following sequence

$$
0 \rightarrow E_{L} \rightarrow \cdots \rightarrow E_{1} \rightarrow E_{0} \rightarrow N \otimes_{R} S \rightarrow 0
$$


is exact in $\operatorname{Mod} S$, where each $E_{i}$ is a Ding projective $S$-module as well as a Ding projective $R$-module, by Lemma 3.3. Because $N_{R} \mid\left(N \otimes_{R} S\right)_{R}$ by Lemma 2.3, we have r. $\operatorname{Dpd}_{R} N \leq n$. Hence r. $g 1 . \operatorname{Dpd}(R) \leq n$, that is r. $g 1 . \operatorname{Dpd}(S) \geq$ r. $\operatorname{gl} \operatorname{Dpd}(R)$.

\section{Acknowledgements}

The research is financed by NSFC (Grant Nos. 11201220, 11326065) and the Key Project of Educational Commission of He'nan Province China (No. 13A110834).

\section{References}

Bonami, L. (1984). On the Structure of Skew Group Rings. Algebra Berichte 48, Verlag Reinhard Fischer, Munichen.

Crawley-Boevey, W. W. (1998). On tame algebras and bocses. Proc. London Math. Soc., 5, 451-483.

Ding, N. Q., \& Chen, J. L. (1993). The flat dimensions of injective modules. Manuscripta Math., 78(2), $165-177$.

Ding, N. Q., \& Chen, J. L. (1996). Coherent rings with finite self-FP-injective dimension. Comm. Algebra, 24(9), 2963-2980. http://dx.doi.org/10.1080/00927879608825724

Enochs, E. E., \& Jenda, O. M. G. (1995). Gorensein injective and projective modules. Math. Z., 220(4), 611-633.

Feng, L. G. (1997). Essential extensions, excellent extensions and finitely presented dimension. Acta Math. Sin. (New Ser.), 13, 231-238.

Gillespie, J. (2010). Model structures on modules over Ding-Chen rings. Homology, Homotopy and Applications, $12,61-73$.

Mao, L., \& Ding, N. Q. (2005). Notes on FP-projective modules and FP-injective modules. Advances in Ring Theory, 151-166.

Passman, D. S. (1977). The Algebraic Structure of Group Rings. New York, London-Sydney: Wiley-Interscience.

Stenström, B. (1970). Coherent rings and FP-injective modules. J. London Math. Soc., 2(2), 323-329.

Wang, Z. P., \& Liu, Z. K. Stability of Ding projective modules.

Xiao, Y. F. (1994). SF-rings and excellent extensions. Comm. Algebra, 22, $2463-2471$. http://dx.doi.org/10.1080/00927879408824971

Xue, W. M. (1996). On almost excellent extensions. Algebra Colloq., 3, 125-134.

Yang, C. H. (2013). Strongly Gorenstein flat and Gorenstein FP-injective modules. Turk. J. Math., 37, $218-230$. http://dx.doi.org/10.3906/mat-1012-549

Yang, G. (2012). Homological properties of modules over Ding-Chen rings. J. Korean Math. Soc., 49(1), 31-47. http://dx.doi.org/10.4134/JKMS.2012.49.1.031

\section{Copyrights}

Copyright for this article is retained by the author(s), with first publication rights granted to the journal.

This is an open-access article distributed under the terms and conditions of the Creative Commons Attribution license (http://creativecommons.org/licenses/by/3.0/). 